\title{
Model of Monitoring and Correction of Tools Flow in a Production System
}

\author{
Magdalena BARBU \\ Transilvania University of Brasov, Romania, magda.n@unitbv.ro \\ Gavrilă CALEFARIU \\ Transilvania University of Brasov, Romania, gcalefariu@unitbv.ro
}

\begin{abstract}
Any factory, whatsoever the product it processes and whatsoever the type of material processing, is vulnerable to internal perturbations, among whom the variation of the tool flux is of major importance. The main issue of tool management within the production system consists in the fact that "at request, any tool, of any type and dimension, must be available within the right time, at the right place" to cast off the tool penury in the system. Preventing vulnerability is achieved through management in real time, which, for eliminating the penury and the surplus, must ensure the minimal safety stock, regulated through managerial decision.
\end{abstract}

Keywords

tool flow, perturbation evaluation, analysis of inputs / outputs

\section{Introduction}

The overall tool number necessary for executing a process, and to a even lesser extent the overall tool number existing within the factory at a given moment does not fulfil both theoretical and practical conditions to constitute in a system.

Therefore, as follows, the tools will be deemed as a flux flowing from the central deposit to the factory towards the departmental zonal deposit, towards the machine local deposits, towards the machines, following the perturbation monitoring track and correcting track (control, selection, regrinding/sharpening, broken tool elimination, set-up, pre-adjustment). This one will bear the name of tool flux.

In the fabrication flux, the tools are completely consumed in processing the finite tools, which makes the tool flux not to have its own outlet and therefore be open and atypical. The input is connected to the input exo-environment of the manufacturing system - the market of tool suppliers and the outlet - to the fabrication flux.

The specialized literature does not offer a systemic analysis upon the input / output of the tools in the structure of the production system, upon the hierarchical arrangement of the tools in the structure of the production system, upon the main perturbations induced by the tools to the production system, upon the interactions of the tools with the main components of the production system.

The flow of tools is defined as an atypical flow. The perturbations caused by the flow of tools in the manufacturing systems, as well as their apparition causes are defined and classified.

Monitoring management tools consists in pursuing over the system and in and supervising their consumption almost on every machine throughout system, their spatial circulation on every trajectory of the itinerary transport plan and signalling the penury apparition danger for every tool in the system. Monitoring implies periodically elaborating monitoring reports and sending them over to the tool provisioning department and to the production planning department.

\section{Perturbations in the Flow of Tools}

Perturbations are endo and exogenous phenomena whose effect consists in the undesired output modification when the input command (decision) is not modified. The perturbation causes are endo and exogenous and consequently the perturbations will be endo and exogenous [1]. The endogenous causes of the perturbations are: subjectivism in monitoring and management, utilization of natural technologies in processing, blockages, adaptation process and the exogenous causes are: relations with the input and output exo-environments. Consequently, the perturbations are unpredictable, unstable 
and impossible to avoid, which raises the production systems vulnerability.

For the flow of tools the main endogenous perturbations are: wear and tear, break-up, setting, tool pre-adjustment, non-observance of the process technological parameters, flow of tools organization, tool penury along the flow, absence of the standard piling up tool stock, and the exogenous ones are: unstable relations with the tool suppliers, the manner of organizing the tool provisioning, which are, in general, managerial causes.

The internal tool consumption is a perturbation as it influences the outlets for stationary inputs; the consumption is brought about by the causes: break-ups, wear and tear, derangements denominated as follows tool flaw. If the wear and tear are predictable and therefore may be foreseen in anticipation, with correction effect, through preventive programs, the breaking up and the deregulations are preeminently unpredictable, with decisive influence upon the variation of the quantitative level of the tool flux. The wear and tear only influence the local hierarchical level of the tools, in great number and with relatively great frequency.

Through preventive programs, the influence of the wear and tear upon the level of the tool flux may be reduced up to zero value. The breaking up; and the derangements influence all hierarchical levels: central, zonal and local, bringing along the tool circulation along the entire flux. Although the number of the breaking-ups, of the derangements as well as their frequency is small, comparatively to the wear and tear, their influence upon the variation of the quantitative level of the tool flux is of significance. The tear and wear bring along the tool circulation, exclusively in the endo-environment, between the local piling up stock - tool dresser for re-grinding/sharpening and zonal piling up stock - local piling up stock for replacement, without affecting the quantitative level of the tool flux. The derangements bring along the tool circulation, exclusively within the endo-environment, between the local piling up stock - post of pre-adjustment for the adjustment and the zonal piling up stock - local piling up stock for replacement, without affecting the variation of the quantitative level of the tool flux. The breaking ups induce the tool circulation in the endo-exo-environment and its reverse.

The endo-exo-environment motion results in eliminating the broken tool, as waste, between the local piling up stock and the post for collecting the waste from the exo-environment and the circulation exoendo-environment consists in provisioning from the exo-environment, the central deposit, followed by its motion, set-up and pre-adjusted, through the zonal piling up stock, in the local piling up stock. Consequently, the tool breaking up affects the variation of the quantitative level of the tool flux. The complete utilization of each tool, after a predictable number of re-sharpening operations, influences the quantitative level of the tool flux. Consequently, the provisioned tool quantity, on the inlet, modifies the quantitative level of the tool flux in the endo-environment. Quantity supplied on entry into the central warehouse is the result you want to cancel the disturbance of endo-environment - breaking and complete exhaustion of tools in manufacturing. The quantity of provisioned tools depending on the breaking up, and likewise tear and wear of the tools, unpredictable and consequently unforeseeable, must be managed (monitored) in real time being unpredictable.

\section{Evaluation of Perturbations}

To the purpose of optimizing the performance functionals, there must be known both the perturbations and the blockage causes, as well as their quantitative and qualitative evaluation, which should allow the minimization of their influence upon the performance functionals [2].

The perturbations produce auxiliary times which negatively influence the performance functional. From this standpoint they are undesired. However, some of them as: settings, pre-adjustments pertaining to the adaptation process; control which is a necessary process for the quality of production; sharpening, necessary for the complete utilization of the tools, are needful for optimizing the material processing.

When a parameter of the process is liable to asynchronisms, it is a source of unavoidable but also necessary perturbations during the processing; asynchronism itself is the measure of perturbation.

Production diversity imposes a real technology variable in time, which in its turn requires a variable structure of SFa and consequently a diversified FISc. SP identification and controllability impose the orderly development of all processes, with the observance of the technological precedence conditions 
and of the restrictions in the relations of equipotency, coordination / subordination, which entails priorities among the different production activities. This way, the modification of the orderly range of the technological operations in the process implies the modification, the change of the transport trajectories of the semi-product in the processing, the tool change, the modification of the stocks on the flow, of the utilization degree of the manipulation and storage facilities for Sf/Pf and tools, of the tool programming, of the production capacities and of the manipulation and storage facilities. The greatest effect of these modifications pre-eminently manifests itself in the transitory regime, whose duration must be minimized.

\section{Model of Monitoring and Correction of Tools Flow}

The tactical management of the production breaks down the production plan of the factory into partial manufacturing tasks by sections and the operational management takes them and breaks them down on workshops, manufacturing lines and jobs, for certain intervals (day, decade, month, etc.) from which the production planning documents result [3]. Therefore, the manufacturing preparation compartment determines the necessary tools and the existing tools from the previously known inventory, transmitting to the material supply compartment the necessary supply of new tools from the suppliers, in accordance with the following production plan. Through the production scheduling compartment, both the supply of new tools from the suppliers and the supply of tools are programmed, by redistributing them in the environment, to all the compartments of the production system, avoiding the states of excess and shortage in each component.

The method of advancement provides the necessary tools at the right workplace, at the right time, before launching the production into manufacturing. To specify the process of monitoring the flow of tools in a factory it becomes necessary to specify some notions.

One of the initial conditions for starting the manufacture of a product is the existence of resources, among which, the level of tools needed, qualitatively and quantitatively diversified and the organization of their distribution in the manufacturing system. The flow of tools is atypical because: although it is a material flow, it is independent having its own distinct trajectories relative to the flow of the raw material being processed; it is consumed entirely in the processing of the raw material and therefore has no own output.

Tools are consumable objects in the material processing of the finished part; his participation in the material processing is synergistic, his evaluation being implicit in the finished piece; even in the case of the manufacture of a product, the flow of tools is diversified, depending on the operation, process and type of the product. For a factory that processes a class of stable strategic products over a period, a deterministic diversity of tool flow can be defined, the variation of which is insignificant, which allows to forecast the consumption of tools with a high probability [4]. Depending on its own durability, the tool is consumed in many cycles, being one of the material resources reusable by reassembly and therefore recirculating the manufacturing system. In an irrational management of the flow of tools, phenomena of surplus or shortage can occur.

Due to the particularities of the tool flow, listed above, the manufacturing system requires the storage of tools at all three levels: local, area and central. The entry of tools into the central warehouse will be called the supply of tools (from suppliers). Tool entries in the local and local warehouses will be called the tool feed from the immediately higher-grade storage. The only active output of the tool flow is the local warehouses to the workstations that produce the material processing in which the tool participates.

The attributes of operational management include programming and monitoring the tools flow.

The operating management process is subject to the following disruptions of the tool flow: variation of tool diversity; variation of the dimensions of the same type of tool; tool wear and retrofitting; tool deregulation / adjustment; variable levels of tool stocks of the same type and different tools; the depletion of the tool stock in any stocking and the danger of the tool shortage; the surplus of new tools, unused over a period of time; supplying / replenishing with new tools; power supply / refuelling with new and used tools; the multitude of tool transport routes and the random need to select them.

Consequently, tool flow monitoring is the process that aims to evaluate the status of tools of any kind 
in material processing, the existence of a minimum level stock in any tool called standard stock, evaluating the stock error and transmitting the tool flow status report with a frequency by default, to the programming department. The stages of monitoring are: measurement, data processing, error evaluation, drafting the status report, sending it to the programming department [5].

On the output of the existing system is expressed by $w_{\text {ex }}(k)$, the necessary by $w_{\text {nec }}(k+1)$ and the desired by $\mathrm{w}_{\mathrm{d}}(\mathrm{k}+2)$.

The disturbances in the endo-medium and the exo-medium produce consumption of stock, delivery on the flow, unwanted deviations on the flow so that, always, there is the difference $\left(w_{\mathrm{ex}}-\mathrm{w}_{\mathrm{nec}}<0, \mathrm{w}_{\mathrm{ex}}\right.$ $<W_{\text {nec }}$ - by consumption from the existent) called correction error $\left(-\varepsilon_{\text {cor }} w\right)$ which, in order to continue material processing, must be corrected at the next moment $(\mathrm{k}+1)$, by adding an amount $\left(+\varepsilon_{\text {adw }}(\mathrm{k}+1)\right)$ until the next state is obtained $x(k+1)=w_{d}(k+1)-$ desired. Generally, $w_{d}>w_{n e c}$, when $\left(\left|+\varepsilon_{\text {ad }}\right|=\mid-\right.$ $\varepsilon_{\text {corw }} \mid$ ).

The process of correcting the lack of matter, through a new addition (ad) until the desired level is reached, is called the correction process which is discrete, dynamic, and real-time.

The correction process consists of:

1) evaluation of the correction error by monitoring,

$$
-\varepsilon_{\text {cor } w}(k+1)=w_{\text {ex }}(k)-w_{\text {nec }}(k+1)
$$

2) correction decision

$$
\mathrm{DCor}(\mathrm{k}+2) \rightarrow-\varepsilon_{\text {cor w }}(\mathrm{k}+2) \rightarrow \mathrm{w}_{\mathrm{d}}(\mathrm{k}+2)
$$

3) supply decision

$$
\mathrm{DAp} / \mathrm{Al}(\mathrm{k}+3) \rightarrow+\varepsilon_{\text {cor w }}(\mathrm{k}+2)=\mathrm{NAp} / \mathrm{Al}(\mathrm{k}+3)
$$

4) scheduling of material supply with tools

$$
\left|+\varepsilon_{\text {ad w }}(\mathrm{k}+4)\right|=\left|-\varepsilon_{\text {cor w }}(\mathrm{k}+2)\right| \rightarrow\left(\varepsilon_{\text {cor w }} \rightarrow 0\right) \rightarrow \mathrm{w}_{\mathrm{d}}(\mathrm{k}+4) \geq \mathrm{w}_{\text {nec }}(\mathrm{k}+1)
$$

The correction process being discrete, dynamic and real-time occurs according to the dynamic equation:

$$
\mathrm{x}_{\mathrm{d}}(\mathrm{k}+1)=\mathrm{x}_{\mathrm{ex}}(\mathrm{k})+\mathrm{u}\left(\varepsilon_{\text {cor } \mathrm{x}}(\mathrm{k})\right)=\mathrm{x}_{\mathrm{ex}}(\mathrm{k})+\mathrm{x}_{\mathrm{ad}}\left(\varepsilon_{\text {cor } \mathrm{x}}(\mathrm{k})\right) \geq \mathrm{y}_{\text {nec }}(\mathrm{k}+1)
$$

or

$$
\left.\mathrm{w}_{\mathrm{d}}(\mathrm{k}+1)=\mathrm{w}_{\mathrm{ex}}(\mathrm{k})+\varepsilon_{\text {cor } \mathrm{w}}(\mathrm{k})\right) \rightarrow \mathrm{w}_{\text {nec }}(\mathrm{k}+1)
$$

This expresses the fact that in order to reach the level $\mathrm{w}_{\mathrm{d}}(\mathrm{k}+1) \geq \mathrm{w}_{\mathrm{nec}}(\mathrm{k}+1)$ it is necessary the correction decision in the higher hierarchical level which, for its execution, issues the command $\mathrm{u}\left(\varepsilon_{\text {cor } w}\right)$ based on which, through the process of supply / supply, the level $\mathrm{w}_{\mathrm{d}}=\mathrm{w}_{\text {nec }}$ is obtained.

As a result, the number of variables to be monitored will be equal to the sum of the products between the partial diversity and the partial level of the flux stocks, that is:

$$
\mathrm{s}_{\mathrm{t}} \overline{\mathrm{n}}_{\text {ScsSTC }}+\mathrm{s}_{\mathrm{zt}} \overline{\mathrm{n}}_{\text {ScsSTZ }}+\mathrm{s}_{\mathrm{lt}} \overline{\mathrm{n}}_{\text {ScsSTL }}
$$

in which $\bar{n}_{\text {Scs }}$ they express the average number of tools of the same type $(s=c t$.) from each type of hierarchical stock (C, Z, L) or expressed by the pairs of parameters (diversity, level) to monitor:

$$
\left(s_{t}, N_{\text {STC }}\right)+\left(s_{z}, N_{\text {STZ }}\right)+\left(s_{l}, N_{\text {STL }}\right)
$$

Monitoring is performed simultaneously on all three hierarchical levels $(\mathrm{C}, \mathrm{Z}, \mathrm{L})$, with two variables per level, resulting in six pairs variables and therefore a monitoring scheme of the third order $(\mathrm{C}, \mathrm{Z}, \mathrm{L})$ is required for monitoring two parameters $(\mathrm{s}, \mathrm{N})$.

According to the above rules, the general dynamic equations of the global flow of tools from the previous figure will be:

$$
\begin{aligned}
\mathrm{x}_{\mathrm{si}}(\mathrm{k}+1) & =\mathrm{x}_{\mathrm{si}}(\mathrm{k})+\left(\mathrm{Tc}_{\mathrm{i}}+\mathrm{Tp}_{\mathrm{i}}\right) R p_{\mathrm{i}}(\mathrm{k}) \\
\mathrm{x}_{\mathrm{Ni}}(\mathrm{k}+1) & =\mathrm{x}_{\mathrm{Ni}}(\mathrm{k})+\left(\mathrm{Tc}_{\mathrm{i}}+\mathrm{Tp}_{\mathrm{i}}\right) R p_{\mathrm{i}}(\mathrm{k})
\end{aligned}
$$


and those of a storage (node), taken in isolation, will be:

$$
\mathrm{x}_{\mathrm{si}}(\mathrm{k}+1)=\mathrm{x}_{\mathrm{si}}(\mathrm{k})+\left(\mathrm{Tc}_{\mathrm{i}}+\mathrm{Tp}_{\mathrm{i}}\right)\left[\operatorname{Rac}_{\mathrm{i}}(\mathrm{k})-\operatorname{Rliv}_{\mathrm{i}}(\mathrm{k})\right]
$$

wherein i = STC, STZ, STL, and:

$$
\begin{gathered}
\operatorname{Rp}_{\mathrm{i}}(\mathrm{k})=\frac{\left(\mathrm{x}_{\text {sdi }}(\mathrm{k}), \mathrm{x}_{\mathrm{Ndi}}(\mathrm{k})\right)}{\mathrm{Tc}_{\mathrm{i}}+\mathrm{Tp}_{\mathrm{i}}} \\
\operatorname{Rac}_{\mathrm{i}}(\mathrm{k})=\frac{\left(\mathrm{x}_{\text {sdaci }}(\mathrm{k})-\mathrm{x}_{\text {sexi }}(\mathrm{k})\right),\left(\mathrm{x}_{\mathrm{Ndaci}}(\mathrm{k})-\mathrm{x}_{\text {Nexi }}(\mathrm{k})\right)}{\mathrm{Tc}_{\mathrm{i}}+\mathrm{Tp}_{\mathrm{i}}} \\
\operatorname{Rliv}_{\mathrm{i}}(\mathrm{k})=\frac{\left(\mathrm{x}_{\text {sexi }}(\mathrm{k})-\mathrm{x}_{\text {sdlivi }}(\mathrm{k})\right),\left(\mathrm{x}_{\text {Nexi }}(\mathrm{k})-\mathrm{x}_{\text {Ndlivi }}(\mathrm{k})\right)}{\mathrm{Tc}_{\mathrm{i}}+\mathrm{Tp}_{\mathrm{i}}}
\end{gathered}
$$

The notations have the following meaning: Rc - the ordering rate, Tc - the duration of the order, $\mathrm{Rp}$ - the rate of receipt of the tools from the suppliers, $\mathrm{Tp}$ - the duration of the receipt of the tools from the suppliers, ad - addition (additivity).

\section{Conclusions}

The purpose of the paper was to establish a mathematical model of the tool flow correction process so that it can adapt in real time to the variable conditions and dynamic perturbations of the global production system. By correcting the flow of tools, the necessary tools are provided at the right place of work, at the right time, before launching the production into manufacturing.

The main internal perturbations in the tool management process are the tool penury and excess. Both perturbations make manufacturing system excess vulnerable to the market requirements.

Tools penury may appear at the machine, on the flow, in the zonal section warehouse or in the central factory warehouse. This is a major perturbation which must be eliminated through managerial measures. To this purpose, the tool global administration, including the automated evidence and monitoring is an issue of the tactic management (planning, programming, monitoring department).

During material processing tools are worn and broken; these ones constitute main perturbations in administering tools; for their correction, tools undergo off-line or on-line control, and the used and broken tools are sent to a selection post for worn tool reconditioning / broken tool elimination.

The tool management process fits within the global manufacturing process as Integrated Kanban Process.

\section{References}

1. Olteanu F.C., Gheorghe C. (2016): Aspects regarding the qualitative analysis of risks due to the occurrence of low probability and very high impact events. Review of the Air Force Academy. Available at: http://www.afahc.ro/ro/revista/2016 1/Olteanu Gheorghe 2016 1.pdf

2. Barsan-Pipu N., Popescu I. (2003): Risk Management: Concepts, Methods, Applications. Transilvania University Press, ISBN 973-635-180-7 (in Romanian)

3. Morar L., Vestkamper E., Abrudan I., Pîslă A., Niemann J., Manole I. (2007): Planning and operation of production systems. Fraunhofer IRB, ISBN 978-3-8167-7327-6

4. Zaharia, M., Salagean L., Staicu D.F. (2002): Quantitative methods in decision making. Ecran Magazin Publishing House, Brasov, ISBN 973-8281-08-3 (in Romanian)

5. Lupulescu N.B. (2001): Integration of milling tools in flexible manufacturing systems. Transilvania University Publishing House, Brasov, ISBN 973-8124-54-9 (in Romanian) 